\title{
Volume Modulation of Nonlinear Optical Properties by Cation Substitution
}

\author{
Jingmei Min, ${ }^{\#, \dagger}$ Hao Li, ${ }^{\#,+, \xi}$ and Jun Zhang ${ }^{*}+\dagger$
}

$\uparrow$ School of Physics Science and Technology, Xinjiang University, Urumqi, Xinjiang 830046, China

†CAS key Laboratory of Functional Materials and Devices for Special Environments; Xinjiang Technical Institute of Physics \& Chemistry, CAS; Xinjiang Key Laboratory of Electronic Information Materials and Devices, 40-1 South Beijing Road, Urumqi 830011, China

$\S$ Center of Materials Science and Optoelectronics Engineering, University of Chinese Academy of Sciences, Beijing 100049, China

*E-mail: zhangjunxju@163.com

Figure S1. Static second-order nonlinear susceptibilities of $\mathrm{LiInS}_{2}$ convergence test for $\mathrm{k}$ separations and empty band number.

Figure S2. Calculated Hamiltonian energy profiles for $\mathrm{RbInS}_{2}$ (a) and $\mathrm{CsInS}_{2}$ (b) during the MD simulation at $300 \mathrm{~K}$. And phonon dispersion curves of $\mathrm{RbInS}_{2}$ (c) and $\mathrm{CsInS}_{2}$ (d).

Figure S3. The SHG-density of occupied states and unoccupied states for $\mathrm{AInS}_{2}$ series. Table S1. The bond distances and cell volumes for $\mathrm{AInS}_{2}$ series ( $\left.\mathrm{A}=\mathrm{Li}, \mathrm{Na}, \mathrm{K}, \mathrm{Rb}, \mathrm{Cs}\right)$. Table S2. Elastic constants of orthorhombic AInS 2 calculated using DFT.

Table S3. Calculated band gaps, birefringence $(\Delta n)$ and SHG coefficients $\left(d_{\mathrm{ij}}\right)$ for the $\mathrm{AInS}_{2}$ series. 
Figure S1. Static second-order nonlinear susceptibilities of $\mathrm{LiInS}_{2}$ convergence test for $\mathrm{k}$ separations and empty band number.
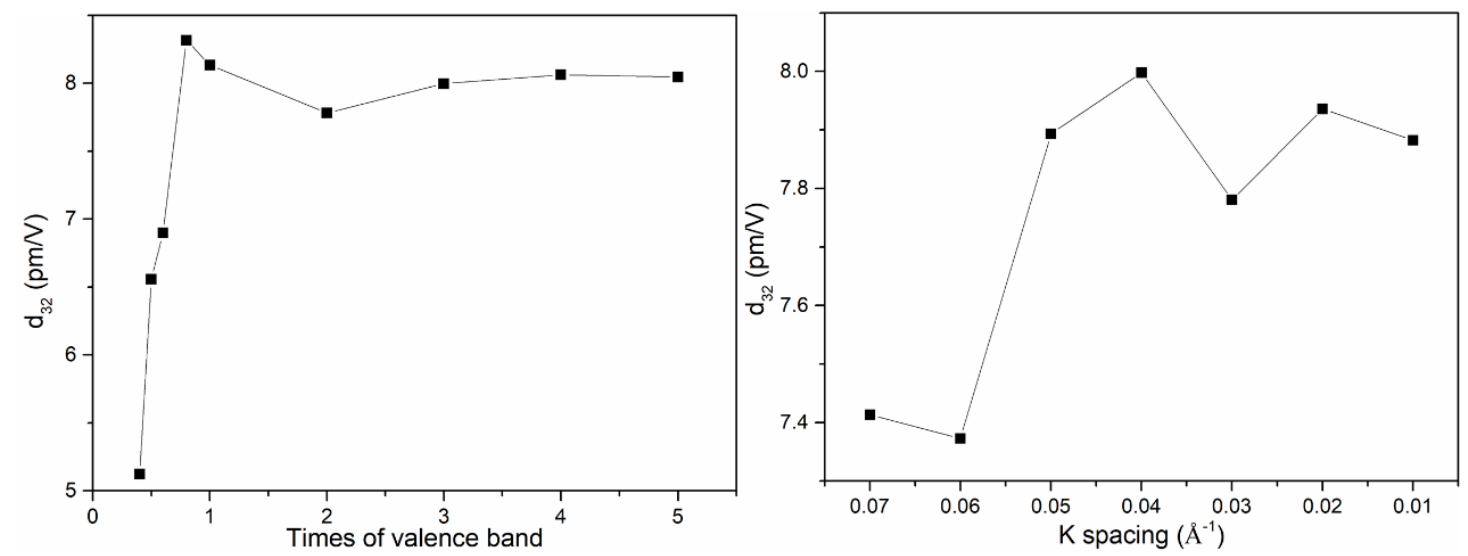

For empty band number, $\mathrm{d}_{32}$ begin to converge at one times of valence band. In the paper, we choose three times of valence band to ensure enough empty bands for the transition.

For $\mathrm{k}$ spacing, $d_{32}$ is converged at $\mathrm{k}$ sample of $0.04 \AA^{-1}$, the corresponding $\mathrm{k}$ mesh is 4 $\times 3 \times 4$ or $\mathrm{LiInS}_{2}$. Denser k separations, including $0.03,0.02,0.01$, not only cannot get better results, but also more expensive. Therefore, for all compounds in this paper, we choose the k spacing of $0.04 \AA^{-1}$. 
Figure S2. Calculated Hamiltonian energy profiles for $\mathrm{RbInS}_{2}$ (c) and $\mathrm{CsInS}_{2}$ (d) during the MD simulation at $300 \mathrm{~K}$. And phonon dispersion curves of $\mathrm{RbInS}_{2}$ (a) and $\mathrm{CsInS}_{2}$ (b).

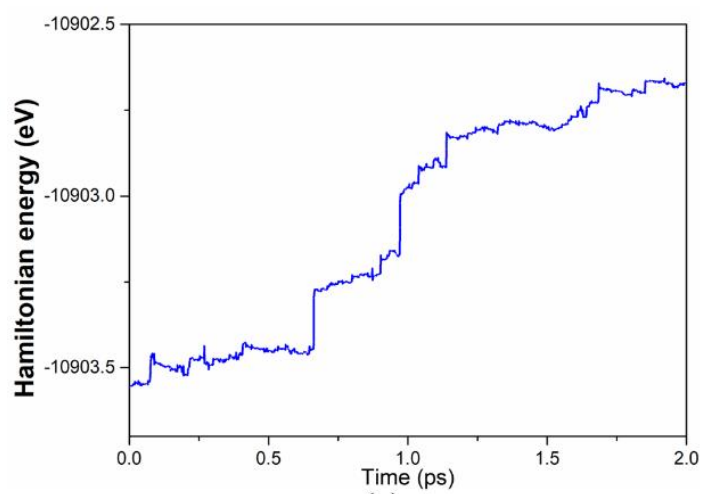

(a)

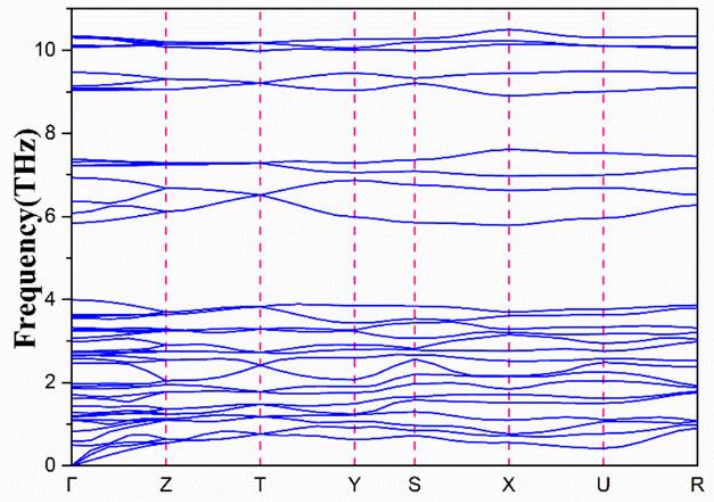

(c)

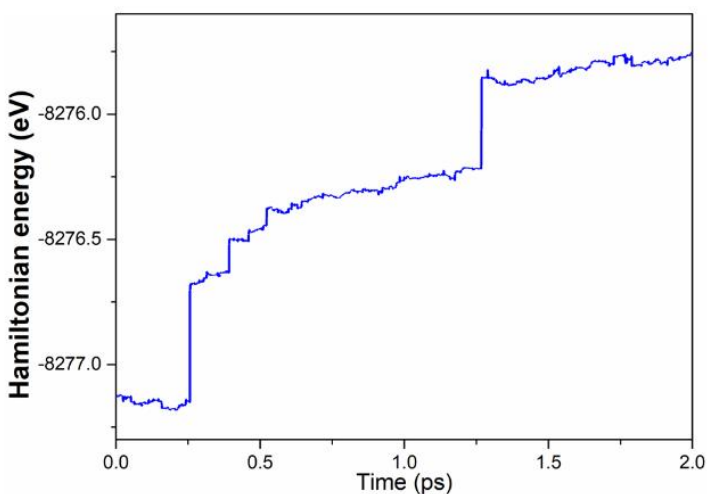

(b)

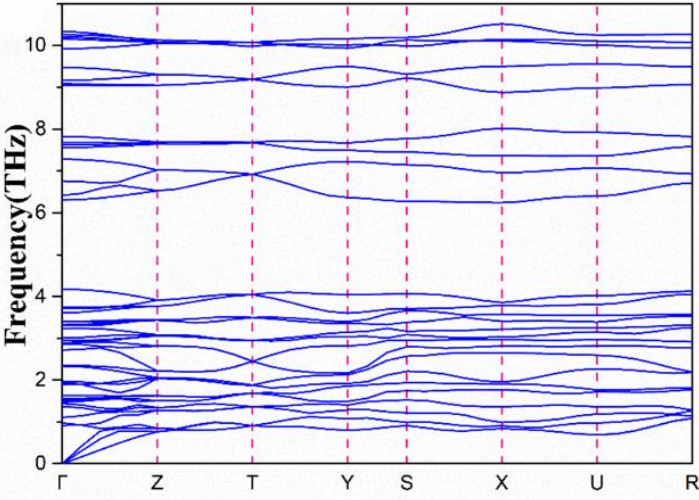

(d) 
Figure S3. The SHG-density of occupied states and unoccupied states for $\mathrm{AInS}_{2}(\mathrm{~A}=$ $\mathrm{Li}, \mathrm{Na}, \mathrm{K}, \mathrm{Rb}, \mathrm{Cs})$
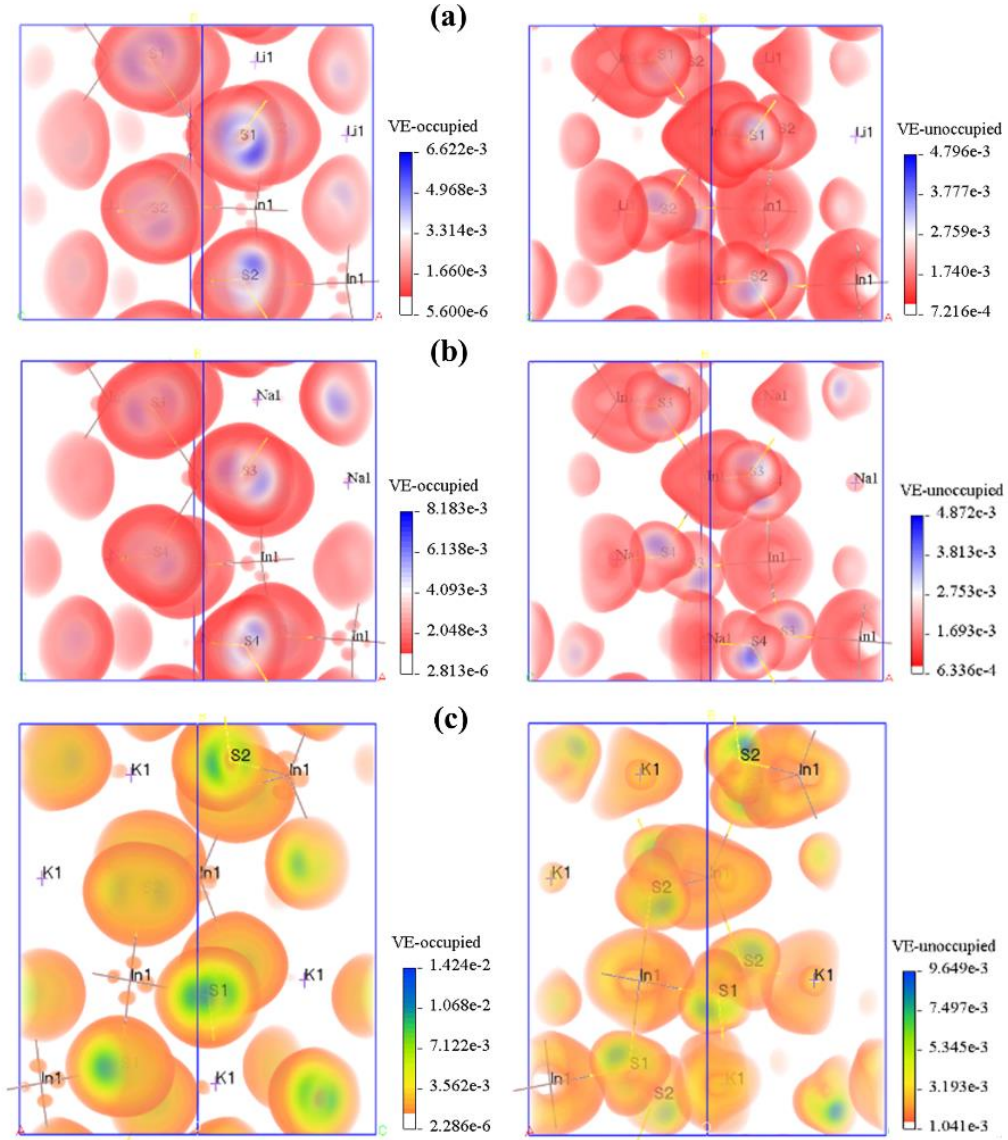

(c)
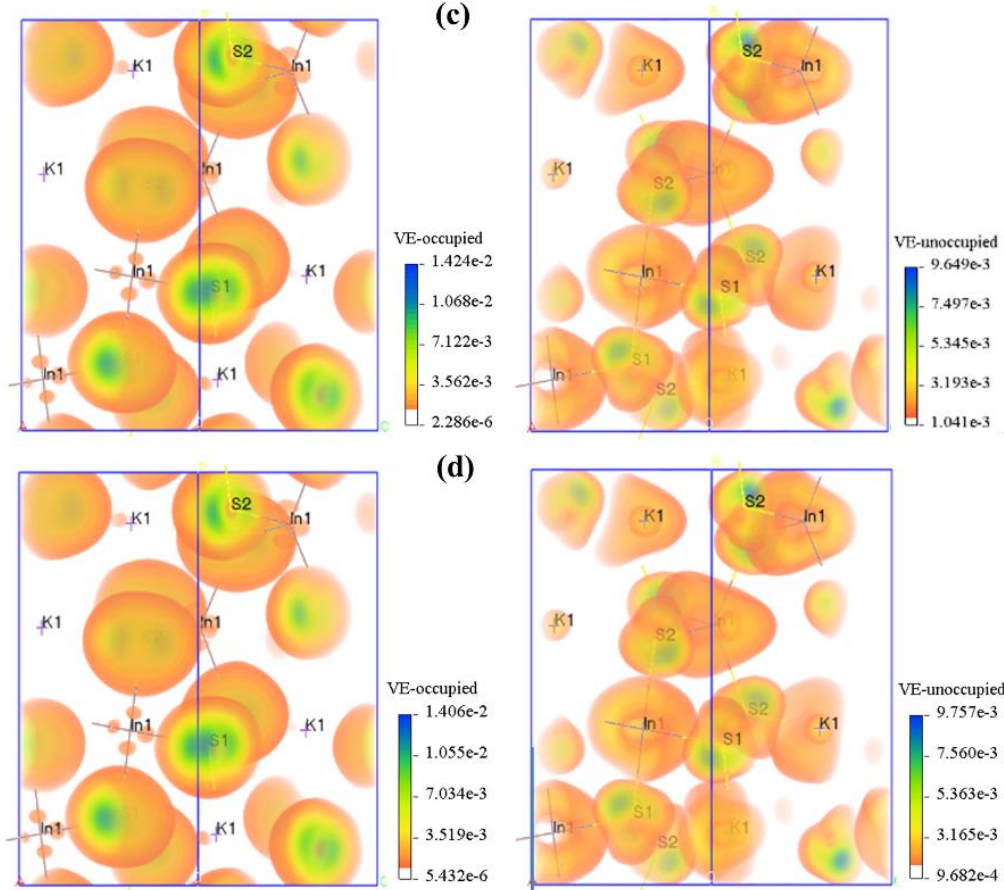

(d)

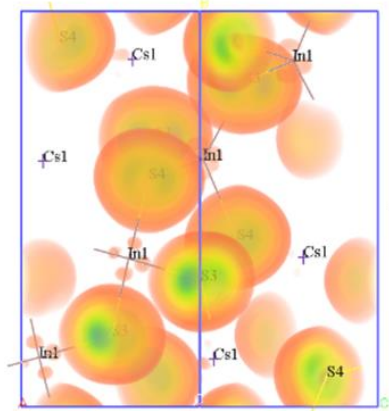

(e)
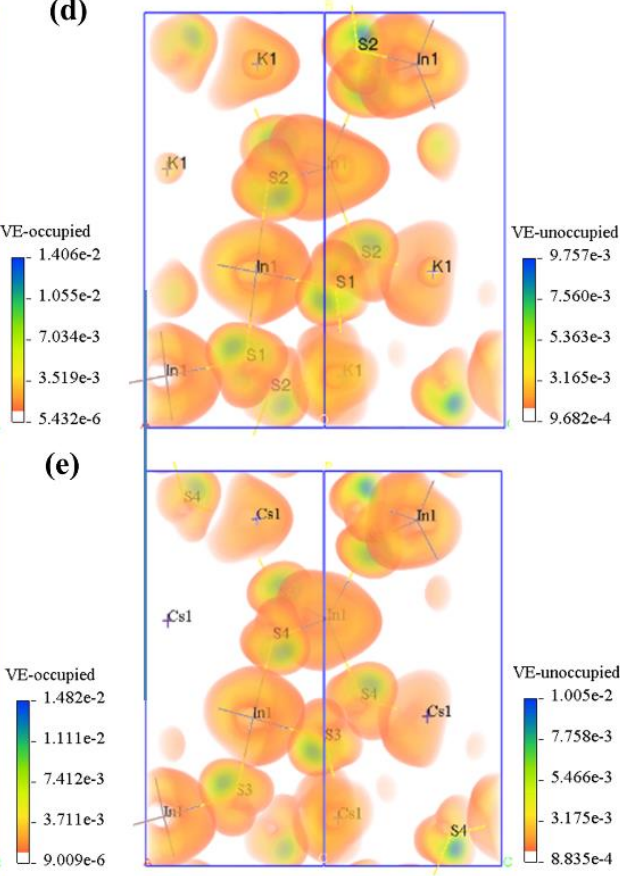
Table S1. The bond distances and cell volumes for $\mathrm{AInS}_{2}$ series.

\begin{tabular}{|c|c|c|c|c|}
\hline Compounds & Species & Bond distances $(\AA)$ & Cell volumes & Structure types \\
\hline \multirow{2}{*}{$\mathrm{LiInS}_{2}$} & In-S & $2.514-2.527$ & \multirow{2}{*}{356.36} & \multirow{4}{*}{$\mathrm{A}^{\mathrm{I}} \mathrm{InS}_{2}$} \\
\hline & $\mathrm{Li}-\mathrm{S}$ & $2.355-2.365$ & & \\
\hline \multirow{2}{*}{$\mathrm{NaInS}_{2}$} & In-S & $2.509-2.526$ & \multirow[b]{2}{*}{452.63} & \\
\hline & $\mathrm{Na}-\mathrm{S}$ & $2.763-2.797$ & & \\
\hline \multirow{2}{*}{$\mathrm{KInS}_{2}$} & In-S & $2.513-2.535$ & \multirow{2}{*}{480.48} & \multirow{6}{*}{$\mathrm{A}^{\mathrm{II}} \mathrm{InS}_{2}$} \\
\hline & $\mathrm{K}-\mathrm{S}$ & $3.154-3.543$ & & \\
\hline \multirow[b]{2}{*}{$\mathrm{RbInS}_{2}$} & In-S & $2.514-2.535$ & \multirow[b]{2}{*}{516.4} & \\
\hline & $\mathrm{Rb}-\mathrm{S}$ & $3.289-3.678$ & & \\
\hline \multirow{2}{*}{$\mathrm{CsInS}_{2}$} & In-S & $2.515-2.537$ & \multirow{2}{*}{565.93} & \\
\hline & Cs-S & $3.468-3.869$ & & \\
\hline
\end{tabular}


Table S2. Elastic constants of orthorhombic $\mathrm{AInS}_{2}$ calculated using DFT.

\begin{tabular}{llllllllll} 
Crystal & $\mathbf{C}_{11}$ & $\mathbf{C}_{22}$ & $\mathbf{C}_{33}$ & $\mathbf{C}_{44}$ & $\mathbf{C}_{55}$ & $\mathbf{C}_{66}$ & $\mathbf{C}_{12}$ & $\mathbf{C}_{13}$ & $\mathbf{C}_{23}$ \\
NaInS2 $_{2}$ & 45.4 & 47.1 & 41.5 & 12.7 & 8.7 & 13.6 & 30.3 & 20.7 & 26.3 \\
\hline $\mathbf{K I n S}_{2}$ & 22.4 & 70.5 & 25.2 & 17.4 & 6.6 & 16.8 & 23.1 & 5.7 & 23.3 \\
\hline $\mathbf{R b I n S}_{2}$ & 24.4 & 64.1 & 25.9 & 15.6 & 4.9 & 15.0 & 23.9 & 7.0 & 22.8 \\
\hline $\mathbf{C s I n S}_{2}$ & 26.8 & 51.9 & 26.7 & 13.6 & 3.3 & 12.6 & 26.2 & 11.1 & 22.5 \\
\hline
\end{tabular}


Table S3. Calculated band gaps, birefringence $(\Delta n)$ and SHG coefficients $\left(d_{\mathrm{ij}}\right)$ for the $\mathrm{AInS}_{2}$ series (A= Li, Na, K, Rb, Cs).

\begin{tabular}{|c|c|c|c|c|c|c|c|}
\hline \multirow{2}{*}{$\begin{array}{c}\text { Compound } \\
\text { s }\end{array}$} & \multicolumn{3}{|c|}{ Band gap (eV) } & \multirow{2}{*}{$\begin{array}{c}\Delta n \\
@ 1064 \\
\text { nm }\end{array}$} & \multicolumn{3}{|c|}{$d_{\mathrm{ij}}(\mathrm{pm} / \mathrm{V})$} \\
\hline & GGA & $\begin{array}{c}\text { HSE0 } \\
6\end{array}$ & Exp. & & $d_{31}$ & $d_{32}$ & $d_{33}$ \\
\hline $\mathbf{L i I n S}_{2}$ & 2.34 & 2.94 & 3.54 & 0.036 & 7.6 & 8.0 & -16.9 \\
\hline $\mathrm{NaInS}_{2}$ & 2.18 & 2.81 & $*$ & 0.022 & 9.7 & 9.0 & -19.0 \\
\hline $\mathrm{KInS}_{2}$ & 2.11 & 2.77 & $*$ & 0.147 & 5.5 & 10.3 & -21.3 \\
\hline $\mathbf{R b I n S}_{2}$ & 2.11 & 2.70 & $*$ & 0.133 & 6.9 & 12.3 & -24.7 \\
\hline $\mathrm{CsInS}_{2}$ & 2.11 & 2.60 & $*$ & 0.102 & 9.1 & 15.4 & -28.5 \\
\hline
\end{tabular}

